\title{
Title: Bone loss with antiepileptic drug therapy: a twin and sibling study
}

\section{Authors}

Baemisla Shiek Ahmad $\mathrm{PhD}^{1,6}$, Sandra Jane Petty $\mathrm{PhD}^{1}$, Alexandra Gorelik MSc ${ }^{1,3}$, Terence John O’Brien $\mathrm{MD}^{1}$, Keith David Hill $\mathrm{PhD}^{4}$, Jemma J Christie B AppSci ${ }^{1}$, Phillip Neil Sambrook MD ${ }^{5 a}$, John Dennis Wark $\mathrm{PhD}^{1,2}$

\section{Institutional affiliations}

${ }^{1}$ Department of Medicine,University of Melbourne,The Royal Melbourne Hospital,Parkville,Victoria 3050 AUSTRALIA

${ }^{2}$ The Royal Melbourne Hospital Bone and Mineral Service,Parkville,Victoria 3050 AUSTRALIA

${ }^{3}$ Melbourne EpiCentre,The Royal Melbourne Hospital,Parkville,Victoria 3050 AUSTRALIA ${ }^{4}$ School of Physiotherapy and Exercise Science,Curtin University,Perth,Western Australia,6845 AUSTRALIA

${ }^{5}$ Department of Medicine,The University of Sydney,Royal North Shore Hospital,New South Wales 2006 AUSTRALIA

${ }^{6}$ Faculty of Medicine,University of Malaya,Kuala Lumpur 50603 MALAYSIA

\section{Contact information for author correspondence}

Professor John D Wark. Department of Medicine, The University of Melbourne, Level 4, Clinical Sciences Building, The Royal Melbourne Hospital, Parkville,Victoria 3050 AUSTRALIA. Phone:+613 93427109; Fax:+613 93482254

\section{E-mail adresses of authors}

Baemisla Shiek Ahmad: bshiekahmad@ummc.edu.my; Sandra Jane Petty:

pettys@unimelb.edu.au; Alexandra Gorelik: alexandra.gorelik@mh.org.au; Terence John O’Brien: obrientj@unimelb.edu.au; Keith David Hill: keith.hill@curtin.edu.au Jemma J Christie: jemma.christie@icloud.com; John Dennis Wark: jdwark@unimelb.edu.au

${ }^{\mathrm{a}}$ Professor Sambrook passed away in 2012 


\section{Manuscript information}

Manuscript word count (excluding title,author information \& abstract): 3,909 ( $\leq 5000)$

Title character count (spaces and punctuation): 67

Number of references: $38(\leq 45)$

Table count: 3

Figure count: 1 ( $\leq 6$ tables/figures)

\section{Disclosures}

This research was funded by National Health and Medical Research Council project grant number (400089), by the Department of Medicine, University of Melbourne and by the Royal Melbourne Hospital Research Foundation. Professors Wark and O’Brien received research support from UCB Pharma and Novartis for unrelated studies. Dr Petty received research support from UCB Pharma for an unrelated investigator-initiated study. Dr Shiek Ahmad obtained financial support throughout her PhD from the University of Malaya and the Malaysian Ministry of Higher Education. Professors Hill and Sambrook, Ms Gorelik and Ms Christie report no disclosures and declare that they have no conflict of interest.

\section{Acknowledgments}

Authors' individual contributions: Professor Wark leads the University of Melbourne Twin and Sibling Bone Health Program and proposed the research design. Professors Wark, O'Brien and Hill supervised the study. Dr Petty initiated the baseline recruitment and contributed part of the preliminary baseline data. Dr Shiek Ahmad conducted the longitudinal observation study, performed data management and wrote the manuscript. Ms Gorelik and Dr Shiek Ahmad performed data analysis. Dr Shiek Ahmad included a chapter based on this study as a part of her PhD thesis submitted in 2012 to the University of Melbourne. Dr Ahmad accepts responsibility for the integrity of the data management and analysis. Professor Sambrook assisted with recruitment at the Royal North Shore Hospital (RNSH). 
Ms Christie supervised participant assessments in Western Australia. All authors contributed to manuscript writing and review.

The authors would like to express their highest appreciation to all participants for their invaluable contributions and commitments throughout the study, our collaborators, Ms Mary Sakellarides who continued the work of Dr Petty on baseline recruitment, the late Ms Sue Kantor, and Ms Ashwini Kale at Bone and Mineral Medicine, the Royal Melbourne Hospital for their assistance during the bone scanning of Melbourne-based participants, Professor Richard L Prince and his research team at the Sir Charles Gairdner Hospital for their support for study visits in Western Australia, Ms Monique Macara from RNSH who conducted the assessments for Sydney-based participants, the Australian Twin Registry and all who have been involved with the University of Melbourne Twin and Sibling Research Program. 


\section{Abstract}

Purpose: To investigate the longitudinal associations of bone mineral measures with antiepileptic drug (AED) use, including enzyme inducing (EIAED) and non-enzyme inducing (NEIAED) types, and other predictors of bone loss in a study of 48 same-sex twin/agematched sibling pairs (40 female, 8 male) discordant for AED use.

Methods: Using dual-energy x-ray absorptiometry (DXA), areal bone mineral density $(\mathrm{aBMD})$ and content $(\mathrm{BMC})$ at the hip regions, forearm, lumbar spine and whole body were measured twice, at least 2 years apart. The mean within-pair difference (MWPD), MWPD\% and mean annual rate of aBMD change were adjusted for age, weight and height. Predictors of bone loss were evaluated.

Results: AED users, compared to non-users, at baseline and follow-up, respectively, had reduced aBMD at the total hip (MWPD\%: 3.8\%, 4.4\%), femoral neck $(4.7 \%, 4.5 \%)$ and trochanter regions $(4.1 \%, 4.6 \%)(\mathrm{p}<0.05)$.

For the whole cohort, the annual rate of change in all aBMD/BMC $(\mathrm{p}>0.05)$ regions did not differ within pairs. Nevertheless, EIAED users had greater aBMD loss than non-users $(n=20$ pairs) at the total hip ( $1.7 \%$ vs. $0.3 \%, \mathrm{p}=0.013)$ and whole body regions $(0.7 \%$ loss vs. $0.1 \%$ BMD gain, $\mathrm{p}=0.019)$, which was not found in NEIAED-discordant pairs $(\mathrm{n}=16)$. AED use $>20$ years predicted higher aBMD loss at the forearm $(\mathrm{p}=0.028)$, whole body $(\mathrm{p}=0.010)$, and whole body BMC $(\mathrm{p}=0.031)$. 
Conclusions: AED users had reduced aBMD at the hip regions. Prolonged users and EIAED users had greater aBMD loss, predicting a higher risk of bone fragility. Further prospective studies of AED effects on bone microarchitecture are needed.

Abstract word count: $250(\leq 250$ words $)$

\section{Key words}

Longitudinal study, bone mineral density (BMD), antiepileptic drug, dual-energy x-ray absorptiometry (DXA), epilepsy ( $\leq 6$ keywords) 


\section{Introduction}

There is a strong association between antiepileptic drug (AED) use and bone disease [1]. However, the few studies investigating the extent of AED effects on bone measures over time have been limited by lack of comparison subjects [2,3], focus on older individuals [4-6], inclusion of only one gender [3,7], and assessment of two or less clinically-important fracture-risk sites [2,7]. Moreover, the effects of different AED types [enzyme-inducing (EIAED) and non-enzyme-inducing (NEIAED)] on bone loss are not fully understood and studies examining these effects have produced conflicting findings $[3,5,8,9]$.

A well-designed longitudinal study has the potential to facilitate our understanding of the time course of bone loss, risk profiles including AED types implicated, and identification of skeletal sites that may be more prone to bone loss with ongoing therapy. This information may help to discern AED users at high risk for adverse bone effects and guide subsequent treatment choices.

This study aimed to investigate the cross-sectional differences and the longitudinal association of chronic AED use with bone mineral measures in a cohort of same-sex twin and sibling pairs who were discordant for AED use, utilizing a statistically-powerful exposurediscordant twin and sibling pair study design [10]. The secondary aims included the examination of associations with AED types (EIAEDs, NEIAEDs) and the predictors of bone loss. 


\section{Methodology}

\section{Protocol approval and patient consent}

Melbourne Health Human Research Ethics Committee (HREC) (HREC 2003.249), Sir

Charles Gairdner HREC, Perth and HARBOUR HREC of Northern Sydney Central Coast Health (NSCCH) (Protocol 0409-199M (Q)) approved this multi-center study. All participants provided written informed consent. The project was approved by the Australian Twin Registry.

\section{Recruitment}

Same-sex AED exposure-discordant twin and sibling pairs ( $\leq 3$ years age difference) aged between 18 and 75 years, who were community-dwelling, ambulatory, able to communicate in the English language and able to give written informed consent were invited to participate. Pairs were recruited through the University of Melbourne Twin and Sibling Bone Health Research Program at the Royal Melbourne Hospital in collaboration with the Department of Rheumatology at the Royal North Shore Hospital (University of Sydney), and the School of Medicine and Pharmacology, Sir Charles Gairdner Hospital (University of Western Australia). Participants were required to undertake bone mineral examinations on two occasions separated by at least a two-year interval.

Some participants were involved previously in a cross-sectional study examining the potential effect of AEDs on bone measures, which was conducted by our group [11]. The current study was designed to extend our previous work. Pairs who had been involved in the earlier study were invited to return for re-assessment. Additional twin and sibling pairs were also recruited. 


\section{Inclusion and exclusion criteria}

The key eligibility criteria were that one member of the pair was taking continuous AED therapy (users), regardless of the clinical diagnosis, for at least for 12 months prior to baseline [11] and from the baseline up to the follow-up assessment (a minimum continuous AED exposure of three years), while the other twin or sibling must never have had AED treatment (non-users).

Exclusion criteria were: prolonged therapy ( $\geq 6$ months) with non-AED medications affecting bone metabolism including glucocorticoids (with the exception of inhaled steroids for asthma), methotrexate or other antineoplastic treatment, gonadotropin-releasing hormone agonists, cyclosporine for autoimmune diseases; chronic active illness (e.g. cancers, liver or kidney disease, malabsorption syndrome); hyperthyroidism; parathyroid disorders; history of bone diseases (e.g. metastatic bone disease, Paget's disease) with the exception of osteopaenia or osteoporosis as these may be related to prolonged AED use, which is relevant to this study. Participants taking anti-resorptive therapies were excluded.

\section{Study location}

Study visits were performed at the Royal Melbourne Hospital (40 pairs), the Sir Charles Gairdner Hospital, Western Australia (4 pairs) and the Royal North Shore Hospital, Sydney (4 pairs).

\section{Data collection and anthropometry measures}

Data collected via questionnaires included demographics, medical and medication history, epilepsy history and AED therapy, family history, lifestyle history, and history of fractures and falls [11]. Anthropometry measurements were taken on each visit. Participant's weight 
was measured on a non-electronic scale (Continental Scale Corporation Pty. Ltd, Bridgeview, Illinois, 60455 USA) to the nearest 0.1 kilograms (kg), and height using a stadiometer (Holtain Ltd, Crymych, Dyfed, SA41 3UF, UK) to the nearest 0.1 centimetres (cm).

\section{Anti-epileptic drug use}

Participants taking any AEDs were categorized as AED users. Users must have had AED treatment for at least 12 months prior to their baseline examination, and been on continuous AED therapy from baseline until the follow-up assessment. At follow-up, users who had commenced a particular AED type (EIAED, NEIAED, or combined EIAED and NEIAED therapy), either one drug or a combination of drugs of the same type, for a minimum of one year were categorized as current users of that AED type. AEDs categorized as enzyme inducers were: carbamazepine, phenytoin, primidone, phenobarbital/phenobarbitone, oxcarbazepine, topiramate and felbamate $[12,13]$. AEDs categorized as non-enzyme inducers were: sodium valproate, gabapentin, lamotrigine, levetiracetam, ethosuximide, tiagabine, pregabalin, clonazepam, vigabatrin, zonisamide, clobazam and acetazolamide [14].

\section{Bone evaluation}

Melbourne pairs were scanned by dual energy x-ray absorptiometry (DXA) using Hologic QDR 4500A ${ }^{\mathrm{TM}}$ (SN 45558, software version 8.26a:3); Sydney and Perth pairs were scanned using Hologic QDR $4500 \mathrm{~W}^{\mathrm{TM}}$ (SN 47492, software version 12.6.1) and Hologic QDR Discovery A (SN 82641, software version 12.6.1), respectively. Measurements were taken in accordance with the manufacturer's technical manual [15].

Daily assessment of the precision of measurements produced by the Hologic DXA machine was performed via a quality control scan using a spine phantom provided by the 
manufacturer. The mean coefficient of variation $(\mathrm{CV})$ demonstrated by the machine was 0.39\% from February 2009 to November 2011 (mean=1.0536 gm/ $\mathrm{cm}^{2}, \mathrm{SD}=0.0041$ ), confirming excellent reproducibility.

To maximize the accuracy of bone measurement and minimize operator error, both siblings were scanned using the same densitometer for all bone regions on the same day and by the same densitometry technician.

The areal bone mineral density (aBMD) in $\mathrm{g} / \mathrm{cm}^{2}$ and content $(\mathrm{BMC})$ in grams were measured at the hip regions (total hip, neck of femur, trochanter, inter-trochanter), lumbar spine (L2-L4), total forearm and whole-body.

\section{Statistical analyses}

Using the online DSS Research sample size calculator, with the preliminary data obtained from 19 pairs during the initial year of the longitudinal study, power calculations indicated 19 pairs were required to demonstrate a possible difference between AED user and non-user groups in the mean annual rate of aBMD change at total hip. For the femoral neck and the spine regions, respectively 65 and 55 pairs were required.

Statistical analyses were performed using IBM SPSS Statistics 23.0 (IBM Corp., Armonk, NY) and Stata Software Version 13.0 (Stata Corp., TX, USA).

Descriptive data were analysed using paired t-tests for parametric data, the Wilcoxon Rank Test for non-parametric data and the Chi-square test for categorical data. Pearson correlations were performed to examine the associations between age, height, weight and bone measures. 
All bone measures were adjusted for age, height and weight prior to analysis. Paired t-tests were utilized to compare the cross-sectional and longitudinal values for each bone regional parameter. Cross-sectional results were reported as mean within-pair difference (MWPD, users minus non-users mean aBMD/BMC) and MWPD\% (mean within-pair difference in aBMD x $100 \%$ / non-users mean aBMD) for each region. Longitudinal data were expressed as the mean annual rate of aBMD change (mean aBMD value at follow-up minus baseline aBMD, divided by the assessment interval in years (calculated to two decimal places) and expressed as an absolute value of $\left.\mathrm{g} / \mathrm{cm}^{2} / y e a r\right)$. The length of interval years between assessments has been calculated to two decimal places based on the real dates, thus allowing conversion of the interval period into total months or weeks.

Multivariate regression analysis was performed to investigate the potential predictors of the annual rate of bone change at each region after adjustment for relevant covariates: AED user status, sex, current use of vitamin D supplementation, daily calcium intake in the past three months (mg/day), menopausal status, current smoking status, concurrent use of multiple AEDs, and AED therapy duration $(\leq$ or $>20$ years, based on the cohort mean AED therapy duration). Participants who were currently using postmenopausal hormone replacement therapy (HRT) were excluded from the multivariate regression analysis.

A p value of $<0.05$ was used to determine significance for all analyses. 


\section{Results}

\section{Study cohort}

From 1999 to 2010, 92 pairs of twins and siblings were recruited for baseline examination. The follow-up examination was performed from 2009 to 2011. Of the 92 pairs, 56 pairs $(61 \%)$ were eligible and returned for follow-up. Thirty-six pairs were either excluded or failed to return, where one or both of the pair had: deceased (2); moved interstate or overseas (3); cancer and chemotherapy (2); become un-contactable (7); failed to attend follow-up (2); declined ongoing participation (10); long-term prednisolone use (1); become pregnant or were breastfeeding (2); diabetes mellitus with chronic renal disease (1); multiple sclerosis on methyl prednisolone (1); commenced taking AED (1); and ceased taking AEDs (4). Although 56 pairs completed follow-up, eight pairs had to be subsequently excluded because: baseline and follow-up bone evaluations were performed on a different DXA Hologic scanner (2); and taking antiresorptive therapy for osteoporosis (6). Forty-eight remaining pairs were included in the final analysis. AED-discordant pairs prescribed postmenopausal HRT (two users and six non-users) were included in the demographic data and within-pair bone analyses, but not the multivariate regression analysis.

\section{Participant profile}

Of the 48 pairs, $40(83 \%)$ were female and $8(17 \%)$ were male. There were $19(40 \%)$ samesex twin pairs (10 dizygotic; 9 monozygotic), while 29 (60\%) were same-sex sibling pairs. Forty-seven pairs (98\%) were of European or Caucasian ethnicity and 1 (2\%) pair was of mixed European-Asian ethnicity. The cohort mean age at baseline was $47(\mathrm{SD}=15)$ years 
(68\% aged 30-59 years). The median interval between visits was 2.6 (IQR=1.3, range 2-12) years.

No significant difference was found between AED user and non-user groups in age, weight, height, body mass index at baseline and follow-up. At follow-up, these groups did not differ in menopausal status, the number of postmenopausal women with and without current HRT use, mean menopausal age, total number of falls in the preceding year, previous fractures, medical and medication history, and other health and lifestyle characteristics $\quad(p>0.05$, Table 1). Five (10\%) pairs had a family history of epilepsy.

\section{AED user profile}

The AED users were mostly epilepsy patients (94\%) with well-controlled seizure activity ( $86 \%$ had two or less seizures in the preceding year). Some users were taking AEDs as a treatment for migraine $(2,4 \%)$ and trigeminal neuralgia $(1,2 \%)$. Focal epilepsy $(48 \%)$ was the most common epilepsy syndrome. The median duration of AED therapy was 18 years (IQR=19, range 2-64). Most users (60\%) were currently treated with a single AED and $42 \%$ were treated with an EIAED (one drug or in combinations). The most common AEDs used (one drug or in combinations) were sodium valproate (40\%), carbamazepine (35\%), lamotrigine $(21 \%)$ and phenytoin $(15 \%)$. Current users were analysed in three categories: EIAEDs (20 users), NEIAEDs (16 users) and combined EIAEDs and NEIAEDs use (12 users).

\section{Areal BMD measures}

At baseline, compared to their matched non-users, AED users had lower aBMD at the total hip ( $\mathrm{p}=0.044)$, femoral neck $(\mathrm{p}=0.030)$ and trochanteric regions $(\mathrm{p}=0.028)$ but not at other 
skeletal sites (Table 2). In female pairs $(n=40)$ at baseline, users were found to have a lower aBMD than their matched nonusers at the femoral neck region only $(\mathrm{p}=0.011)$.

Also, when compared to matched non-users at baseline, EIAED users (20 pairs, median AED therapy duration of $20(\mathrm{IQR}=17)$ years) were found to have lower aBMD at several regions including the total hip (MWPD\%: 5.3\%, $\mathrm{p}=0.026)$, femoral neck $(5.3 \%, \mathrm{p}=0.043)$ and trochanteric $(6.5 \%, \mathrm{p}=0.002)$, and near-significance at the lumbar spine $(5.9 \%, \mathrm{p}=0.062)$.

Similarly at follow-up, AED users had lower aBMD than non-users at the total hip $(\mathrm{p}=0.046)$, femoral neck $(\mathrm{p}=0.042)$ and trochanteric regions $(\mathrm{p}=0.030)$. Female users were found to have a lower aBMD at the total hip $(\mathrm{p}=0.033)$, femoral neck $(\mathrm{p}=0.013)$ and trochanteric regions $(\mathrm{p}=0.027)$ when compared to their paired non-users.

Both at baseline and at follow-up, aBMD/BMC measurements at all regions were not significantly associated with either AED therapy duration or menopausal status ( $p>0.05$, data not shown).

Longitudinally, no significant within-pair differences were found overall between the users and non-users, as well as within the AED-discordant female pairs, in the annual rate of change in aBMD/BMC measures ( $p>0.05$, data not shown). However, EIAED users $(n=20)$ had a greater mean annual rate of BMD loss at the total hip $(1.7 \%$ vs. $0.3 \%, p=0.013$, Figure 1) and whole body regions ( $0.7 \%$ loss vs. $0.1 \%$ bone gain, $p=0.019)$ than their matched twins/siblings, which was not seen in NEIAED users (16 pairs, data not shown). 
Regression analysis found that prolonged therapy duration (>20 years, mean duration of AED therapy=20 years $(\mathrm{SD}=12))$ predicted a higher annual rate of BMD loss at the forearm $(\mathrm{p}=0.028)$ and whole body $(\mathrm{p}=0.009)$, as well as whole body BMC $(\mathrm{p}=0.015$, Table 3$)$.

A preliminary analysis was performed to examine the difference in the annual rate of change in aBMD/BMC measures between users of different AED types. Users of carbamazepine monotherapy ( $\mathrm{n}=11$, EIAED) showed a greater annual rate of BMD loss when compared to users of sodium valproate monotherapy ( $\mathrm{n}=7$, NEIAED) at the whole body region (median [IQR]: -0.0117 [-0.0153 to -0.0031$]$ vs. 0.0053 [-0.003 to 0.0111$], \mathrm{p}=0.035)$. In addition, carbamazepine monotherapy users showed a higher annual rate of BMD loss than their paired non-users at the total hip (median [IQR]: -0.0008 [-0.027 to 0.0028$]$ vs 0.0027 [-0.0011 to $0.0114], \mathrm{p}=0.014)$ and whole body regions $(-0.0117$ [-0.0153 to -0.0031$]$ vs. $0.0037[-0.0014$ to 0.0089 ], $\mathrm{p}=0.013$ ). This was not seen in users of sodium valproate monotherapy when compared to their paired non-users.

\section{Discussion}

This novel study investigating the temporal association of ongoing AED use with bone measures, uncommonly presented in the existing research literature, was designed to utilize the powerful exposure-discordant twin and sibling pair model $[11,16]$, providing partial control for genetic and environmental covariates, thus enhancing the power of the study [10]. The study involves evaluation of bone measures at two different points in time and rates of change in these bone measures. It addresses a research gap regarding the association of ongoing AED therapy with reduced BMD and bone loss at the clinically-important fracture-risk sites, which may be susceptible to changes with long term AED use. 
Recognizing BMD loss as a potential consequence of AED therapy is important, especially when considered in the light of the lower BMD and reduced bone strength in aging men and women [17-19], the higher rate of falls and risk of fractures, the balance impairment that also lead to falls and fractures with age [20,21] and AED use [22-25], all of which are common and disabling in this population.

Cross-sectionally, both at baseline and follow-up, AED users had lower aBMD than matched non-users at the hip regions. At baseline, EIAED users exhibited lower aBMD at the hip regions and a trend towards lower aBMD at the lumbar spine. Longitudinally, no differences were seen in any regions between the combined user and non-user cohorts or for those taking NEIAEDs compared to matched pairs. However, EIAED users were found to have a greater annual rate of BMD loss at the total hip and whole body than non-users. Finally, multivariate regression showed that prolonged AED use predicted higher BMD loss (adjusted for age, height and weight) at the forearm, whole body and whole body BMC, suggesting that the risk of progressive bone deterioration is ongoing even with very long term AED therapy.

Consistent with findings from previous cross-sectional studies [26-28], lower aBMD in the hip regions (total hip, femoral neck, trochanteric) that have differing bone architecture was evident in the present study of a predominantly middle-aged cohort of AED users. The trochanter region is primarily trabecular bone whereas the total hip and femoral neck are predominantly cortical, which is critical for bone bending strength [29]. The observed decrease in BMD at these various sites suggests possibly similar effects of AEDs on both types of bone although investigations into this are limited. Nonetheless, BMD reduction, especially via increased cortical porosity, will reduce the strength of cortical bone [30], consequently increasing the risk of fractures, leading to functional impairments [31], higher mortality [32] and socio-economic implications [33]. However, it cannot be excluded that the 
within-pair differences showing persisting lower aBMD only at the hip regions may have been due to limitations of the study or other factors.

In the present study, similar to the findings of Carbone and colleagues [6], the annual rate of BMD change between the combined user and non-user groups did not differ in the various regions measured. This may suggest that any continuing modest differential bone loss with chronic ongoing AED use was not large enough to be detected. Bone loss could be more apparent with a larger cohort size, a longer interval between assessments [4], in a younger cohort $[3,7]$ or with specific AED types $[3,7]$.

The longitudinal study by Carbone et al., which showed no major effect of AEDs on bone loss, involved only postmenopausal women, including 58 users, 7141 non-users and a time interval between study visits of 3 years [6]. The present study involved $83 \%$ women, of whom $43 \%$ were postmenopausal. Therefore, differences in gender, age, hormonal status and AED subtypes might account for our findings with respect to EIAEDs by comparison with the findings of Ensrud et al. [4,5], and Carbone et al. [6].

Our study demonstrated a higher rate of BMD loss in EIAED users than non-users at the total hip ( $1.7 \%$ vs. $0.3 \%)$ and whole body ( $0.7 \%$ vs. $0.1 \%$ bone gain), which was not seen in those taking NEIAEDs. Ensrud and colleagues [5] examined annual rate of BMD loss in older men and found higher total hip bone loss in EIAED users (0.46 vs. $0.35 \%)$ and in NEIAED users (0.53 vs. $0.35 \%)$ than non-users. However, only the hip subregions were reported and no comparison was provided of the rate of BMD loss between NEIAED and EIAED users, nor between the combined user cohort and non-users. 
A one-year study of the effect of specific AED monotherapy in premenopausal women showed deterioration in femoral neck BMD of $2.6 \%$ per year with the EIAED phenytoin [3].

In the present study, although the association of specific AED monotherapies with changes in bone measures was not primarily investigated, our preliminary analysis showed that users of EIAED carbamazepine monotherapy had a greater annual rate of BMD loss at total hip and whole body regions when compared to paired non-users.

Similarly, in another study by our research group examining bone changes among newlydiagnosed patients with epilepsy, during their initial years of AED use, users of carbamazepine monotherapy had an increased annual rate of BMD loss at the total hip and femoral neck regions when compared to non-users, and a higher rate of BMD loss at femoral neck region compared to users of levetiracetam or valproate monotherapy [34]. Taken together, BMD loss at the hip regions seems to occur early [34], as well as late, in the course of EIAED carbamazepine therapy. Future investigations into this with an adequate sample size are needed.

Our study has several limitations. We utilized convenience sampling to recruit all available and eligible participants within the clinical services and the Australian Twin Registry who met the inclusion criteria. Recruitment of twin pairs registered with the ATR was by mail-out to all potentially-eligible pairs. Participants were mostly female, therefore the findings may not fully represent the broader population of AED users. The attrition rate amounted to 39\% of the original sample, which is a common limitation of longitudinal studies [35]. The final sample size, after drop-outs, did not meet all of the original power calculations such that the data acquired were likely to have been insufficient to detect very small differential rates of change in bone measures. In order to ensure stability of the statistical solution and 
considering the cohort sample size, because there was no significant difference between pairs on other potentially relevant independent variables, including alcohol intake and the level of physical activity (Table 1), these factors were not included in the regression analysis. The relatively short follow-up interval may also have limited the detection of bone changes during the study period. Blood sample analyses to assess the various essential biochemical changes including vitamin D levels at the time of bone measurements, which might help explain the BMD changes, were not performed. Nonetheless, previous cross-sectional work by one of our researchers did not indicate any significant difference in fasting serum levels of 25hydroxyvitamin D, 1,25-hydroxyvitamin D and immunoreactive parathyroid hormone between chronic users of AEDs and their non-user twin/sibling pairs [36]. The current study did not provide specific $\mathrm{CV}$ data for each region to allow calculation of the least significant change (LSC) but, apart from the total hip region, most of the longitudinal BMD differences were too small to have reached the likely LSC. However, the small CV\% during the study period was consistent with excellent precision generally. Participants' weight and height were not part of the study inclusion criteria. However, no significant difference was found in weight, height and body mass index between groups at baseline and follow-up. Anthropometric measurements, including body size and age at puberty of potential AED user subjects compared to their siblings, could help identify any possible altered rates of sexual maturation and physical growth secondary to epilepsy or chronic use of AEDs since childhood. This would also require accurately ruling out other illnesses or other reasons, for example nutrition, as causes for differences between the pairs prior to participation, which would be challenging to identify retrospectively. Data on the prevalence or incidence of fractures during the study period were not examined, but are unlikely to be informative without a larger sample size. 
Future research directions may include repeat investigations of BMD changes in a larger cohort, which would include all age groups, both continuous and intermittent AED users, at various therapy stages, age and sex-matched controls, and a longer observation period with some intermediate measurements. This would better enable determination of the average rate of BMD change, allow characterization of the onset and course of any bone changes, as well as exploring the modifying effects of specific factors influencing bone health. Keeping a record of falls and fractures to correlate with BMD throughout the study period would be clinically useful. Assessment of the potential effectiveness of antiresorptive therapy, vitamin $\mathrm{D}$ and calcium supplementation, either separately or in combination, for ameliorating any adverse longitudinal effects of AEDs on bone would be worthwhile. Also, use of newer scanning technology to allow micro-architectural indices to be measured, focusing separately on trabecular and cortical bone parameters [37] may better quantify bone strength and predict fracture risk in AED users.

In conclusion, the within-pair differences showed a reduction in aBMD at the hip regions in AED users compared to non-users. In addition, users taking EIAEDs (median AED therapy duration of 18 years) had lower aBMD at the hip regions and a higher annual rate of bone loss at the total hip and whole body. Prolonged AED use (>20 years) predicted higher aBMD loss at the forearm, whole body BMD and BMC. Clinicians should be aware of this ongoing risk of bone mineral loss with chronic AED therapy. Patients who are at a higher risk for bone loss should ideally have their BMD assessed periodically while on treatment. Guidelines are needed to inform such monitoring. Future studies including investigation of the possible beneficial effects of antiresorptive therapy or other bone treatments in improving BMD in this population also are indicated. 


\section{Declaration}

The work described in this manuscript is consistent with the Journal's guidelines for ethical publication. "All procedures performed in studies involving human participants were in accordance with the ethical standards of the institutional and/or national research committee and with the 1964 Helsinki declaration and its later amendments or comparable ethical standards". 


\section{References}

1. Pack AM (2004) Antiepileptic drugs and bone disease. Clin Rev Bone Miner Metab 2(2):159-165. doi:10.1385/BMM:2:2:159

2. Kim SH, Lee JW, Choi K-G, Chung HW, Lee HW (2007) A 6-month longitudinal study of bone mineral density with antiepileptic drug monotherapy. Epilepsy Behav 10(2):291-295. doi: 10.1016/j.yebeh.2006.11.007

3. Pack AM, Morrell MJ, Randall A, McMahon DJ, Shane E (2008) Bone health in young women with epilepsy after one year of antiepileptic drug monotherapy. Neurology 70(18):1586-1593. doi: 10.1212/01.wnl.0000310981.44676.de

4. Ensrud KE, Walczak TS, Blackwell TL, Ensrud ER, Bowman PJ, Stone KL (2004) Antiepileptic drug use increases rates of bone loss in older women: A prospective study. Neurology 62(11):2051-2057.

5. Ensrud KE, Walczak TS, Blackwell TL, Ensrud ER, Barrett-Connor E, Orwoll ES (2008) Antiepileptic drug use and rates of hip bone loss in older men: A prospective study. Neurology 71(10):723-730. doi: 10.1212/01.wnl.0000324919.86696.a9

6. Carbone LD, Johnson KC, Robbins J et al (2010) Antiepileptic drug use, falls, fractures, and BMD in postmenopausal women: Findings from the Women's Health Initiative (WHI). J Bone Miner Res 25(4):873-881. doi: 10.1359/jbmr.091027

7. Andress DL, Ozuna J, Tirschwell D et al (2002) Antiepileptic drug-induced bone loss in young male patients who have seizures. Arch Neurol 59(5):781-786. 
8. Pack AM (2003) The association between antiepileptic drugs on bone disease. Epilepsy Curr 3(3):91-95. doi: 10.1046/j.1535-7597.2003.03306.x

9. Nakken KO, Tauboll E (2010) Bone loss associated with use of antiepileptic drugs. Expert Opin Drug Saf 9(4):561-571. doi: 10.1517/14740331003636475

10. Wark JD (2003) Influence of nutrition on bone health: The twin model approach. In: New S, Bonjour JP (eds) Nutritional aspects of bone health, Royal Society of Chemistry, Cambridge, pp 451-461.

11. Petty SJ, Paton LM, O'Brien TJ et al (2005) Effect of antiepileptic medication on bone mineral measures. Neurology 65(9):1358-1365. doi: http://dx.doi.org/10.1212/01.wnl. 0000180910.72487 .18

12. Anderson GD (2004) Pharmacogenetics and enzyme induction/inhibition properties of antiepileptic drugs. Neurology 63(10 Suppl 4):S3-S8. doi: http://dx.doi.org/10.1212/ WNL.63.10_suppl_4.S3

13. Elger CE, Schmidt D (2008) Modern management of epilepsy: A practical approach. Epilepsy Behav 12(4):501-539. doi: 10.1016/j.yebeh.2008.01.003

14. Riva R, Albani F, Contin M, Baruzzi A (1996) Pharmacokinetic interactions between antiepileptic drugs: Clinical considerations. Clin Pharmacokinet 31(6):470-493. doi: 10.2165/00003088-199631060-00005 
15. Hologic (1999) Model QDR® 4500 Fan beam x-ray bone densitometer: Technical manual. Hologic Inc., MA, USA.

16. MacInnis RJ, Cassar C, Nowson C et al (2003) Determinants of bone density in 30- to 65-year-old women: A co-twin study. J Bone Miner Res 18(9):1650-1656. doi: 10.1359/jbmr.2003.18.9.1650

17. Khosla S, Riggs BL (2005) Pathophysiology of age-related bone loss and osteoporosis. Endocrinol Metab Clin North Am 34(4):1015-1030. doi: 10.1016/j.ecl.2005.07.009

18. Ito M, Nakata T, Nishida A, Uetani M (2011) Age-related changes in bone density, geometry and biomechanical properties of the proximal femur: CT-based 3D hip structure analysis in normal postmenopausal women. Bone 48(3):627-630. doi: 10.1016/j.bone.2010.11.007

19. Sambrook PN, Cameron ID, Chen JS et al (2007) Influence of fall related factors and bone strength on fracture risk in the frail elderly. Osteoporos Int 18(5):603-610. doi: $10.1007 / \mathrm{s} 00198-006-0290-\mathrm{z}$

20. Milat AJ, Watson WL, Monger C, Barr M, Giffin M, Reid M (2011) Prevalence, circumstances and consequences of falls among community-dwelling older people: Results of the 2009 NSW Falls Prevention Baseline Survey. N S W Public Health Bull 22(3-4):43-48. doi: 10.1071/NB10065 
21. Gill T, Taylor AW, Pengelly A (2005) A population-based survey of factors relating to the prevalence of falls in older people. Gerontology 51(5):340-345. doi:10.1159/000086372

22. Tromp AM, Smit JH, Deeg DJH, Bouter LM, Lips P (1998) Predictors for falls and fractures in the longitudinal aging study Amsterdam. J Bone Miner Res 13(12):19321939. doi: 10.1359/jbmr.1998.13.12.1932

23. Shiek Ahmad B, Hill KD, O'Brien TJ, Gorelik A, Habib N, Wark JD (2012) Falls and fractures in patients chronically treated with antiepileptic drugs. Neurology 79(2):145151. doi: 10.1212/WNL.0b013e31825f0466

24. Zielińska-Więczkowska H, Muszalik M, Kędziora-Kornatowska K (2012) The analysis of aging and elderly age quality in empirical research: Data based on University of the Third Age (U3A) students. Arch Gerontol Geriatr 55(1):195-199. doi:

10.1016/j.archger.2011.07.011

25. Shiek Ahmad B, Wark JD, Petty SJ et al (2015) Changes in balance function with chronic antiepileptic drug therapy: A twin and sibling study. Epilepsia 56(11):17141722. doi: 10.1111/epi.13136

26. Farhat G, Yamout B, Mikati MA, Demirjian S, Sawaya R, El Hajj Fuleihan G (2002) Effect of antiepileptic drugs on bone density in ambulatory patients. Neurology 58(9):1348-1353. 
27. El-Hajj Fuleihan G, Dib L, Yamout B, Sawaya R, Mikati MA (2008) Predictors of bone density in ambulatory patients on antiepileptic drugs. Bone 43(1):149-155. doi: 10.1016/j.bone.2008.03.002

28. Valimaki MJ, Tiihonen M, Laitinen K et al (1994) Bone mineral density measured by dual-energy x-ray absorptiometry and novel markers of bone formation and resorption in patients on antiepileptic drugs. J Bone Miner Res 9(5):631-637. doi: 10.1002/jbmr.5650090507

29. Holzer G, von Skrbensky G, Holzer LA, Pichl W (2009) Hip fractures and the contribution of cortical versus trabecular bone to femoral neck strength. J Bone Miner Res 24(3):468-474. doi: 10.1359/jbmr.081108

30. Schneider P, Stauber M, Voide R, Stampanoni M, Donahue LR, Müller R (2007) Ultrastructural properties in cortical bone vary greatly in two inbred strains of mice as assessed by synchrotron light based micro- and nano-CT. J Bone Miner Res 22(10):1557-1570. doi: 10.1359/jbmr.070703

31. Osnes EK, Lofthus CM, Meyer HE et al (2004) Consequences of hip fracture on activities of daily life and residential needs. Osteoporos Int 15(7):567-574. doi: $10.1007 / \mathrm{s} 00198-003-1583-0$

32. Trombetti A, Herrmann F, Hoffmeyer P, Schurch MA, Bonjour JP, Rizzoli R (2002) Survival and potential years of life lost after hip fracture in men and age-matched women. Osteoporos Int 13(9):731-737. doi: 10.1007/s001980200100 
33. Wiktorowicz ME, Goeree R, Papaioannou A, Adachi JD, Papadimitropoulos E (2001) Economic implications of hip fracture: Health service use, institutional care and cost in Canada. Osteoporos Int 12(4):271-278. doi: 10.1007/s001980170116

34. Shiek Ahmad B, O'Brien TJ, Gorelik A, Hill KD, Wark JD (2016) Bone mineral changes in epilepsy patients during initial years of antiepileptic drug therapy. J Clin Densitom 19(4):450-456. doi: 10.1016/j.jocd.2016.07.008

35. Patel MX, Doku V, Tennakoon L (2003) Challenges in recruitment of research participants. Adv Psychiatr Treat 9(3):229-238. doi: 10.1192/apt.9.3.229

36. Petty SJ, Hill KD, Haber NE, Paton LM, Lawrence KM, Berkovic SF, Seibel MJ, O'Brien TJ, Wark JD (2010) Balance impairment in chronic antiepileptic drug users: A twin and sibling study. Epilepsia 51(2):280-288. doi: 10.1111/j.1528-1167.2009.02254

37. Prevrhal S, Meta M, Genant HK (2004) Two new regions of interest to evaluate separately cortical and trabecular BMD in the proximal femur using DXA. Osteoporos Int 15(1):12-19. doi: 10.1007/s00198-003-1500-6

38. Angus RM, Sambrook PN, Pocock NA, Eisman JA (1989) A simple method for assessing calcium intake in Caucasian women. J Am Diet Assoc 89(2):209-214. 
Figure 1 The annual rate of BMD change at total hip - comparison of values for EIAED user and non-user for each pair

The scatter plot shows the rate of BMD change per annum in $\mathrm{g} / \mathrm{cm}^{2}$ for enzyme-inducing AED (EIAED) users (Y-axis) and their paired non-users (X-axis). Each point represents a twin pair. A negative value at the axis indicates an annual BMD deterioration while a positive value indicates an increase in BMD. The annual changes per individual are the difference in BMD at baseline and follow-up visits divided by the interval years (fractional to two decimal places). Points on the line of equality denote no difference in the annual rate of BMD change between pairs. In this figure, the larger number of points below the line of equality indicates that more of the AED-users have a greater BMD deterioration per annum than their paired non-users (mean-within pair difference in BMD loss $\left.(95 \% \mathrm{CI})=0.012(0.02,0.003) \mathrm{g} / \mathrm{cm}^{2}\right)$. Analysis performed using univariate paired t-test. 
Table 1: Cohort descriptives, lifestyle and health characteristics

\begin{tabular}{|c|c|c|}
\hline \multirow{2}{*}{ Baseline } & AED users & Non-users \\
\hline & \multicolumn{2}{|c|}{ Mean (SD) } \\
\hline Age (years) & $47(15)$ & $47(15)$ \\
\hline Weight $(\mathrm{kg})$ & $69(13)$ & $72(14)$ \\
\hline Height (m) & $1.65(0.1)$ & $1.66(0.1)$ \\
\hline Body Mass Index $\left(\mathrm{kg} / \mathrm{m}^{2}\right)$ & $25(4)$ & $26(5)$ \\
\hline \multicolumn{3}{|l|}{ Follow-up } \\
\hline Falls in the preceding 12 months (n fallers, \%) & $12(25)$ & $6(13)$ \\
\hline Previous fracture/s at any site $(\mathrm{n}, \%)$ & $24(50)$ & $22(46)$ \\
\hline Calcium intake (mg/day) ${ }^{\mathrm{a}}$ & $788(467)$ & $704(335)$ \\
\hline Post-menopausal (n, \%) & $17(35)$ & $17(35)$ \\
\hline Age at menopause (mean years, SD) & $48(5)$ & $47(7)$ \\
\hline Postmenopausal, current HRT use $(\mathrm{n}, \%)^{\mathrm{b}}$ & $2(4)$ & $6(13)$ \\
\hline $\begin{array}{l}\text { Postmenopausal, duration current HRT use (med years, } \\
\text { IQR) }\end{array}$ & $6(0)$ & $8(13)$ \\
\hline Physical activity & & \\
\hline
\end{tabular}




\begin{tabular}{|c|c|c|}
\hline Human Activity Profile Maximum Activity Score & $81(10)$ & $85(8)$ \\
\hline Human Activity Profile Adjusted Activity Score & $77(13)$ & $82(11)$ \\
\hline$\geq 4$ hours any sporting activities per week (n, \%) & $7(15)$ & $10(21)$ \\
\hline$\geq 4$ hours of regular walking per week (n, \%) & $20(42)$ & $15(31)$ \\
\hline Alcohol intake (number of standard drinks/month) ${ }^{c}$ & \multicolumn{2}{|c|}{ med (IQR) } \\
\hline Beer (210ml per drink) & $3(10)$ & $4(4)$ \\
\hline Wine (120ml per drink) & $7(11)$ & $13(18)$ \\
\hline Spirit (mixed drinks, per glass) & $2(4)$ & $4(7)$ \\
\hline Smoking history & \multicolumn{2}{|c|}{ n participants $(\%)$} \\
\hline Ever smoker & $16(33)$ & $17(35)$ \\
\hline Current smoker & $7(15)$ & $7(15)$ \\
\hline Lifetime smoking in total pack-years & $9.6(10)$ & $10.1(8)$ \\
\hline Current medical illness, use of non-AED medications & \multicolumn{2}{|c|}{ n participants $(\%)$} \\
\hline Osteoporosis & $1(2)$ & $0(0)$ \\
\hline Asthma & $7(15)$ & $14(29)$ \\
\hline Hypercholesterolemia & $8(17)$ & $4(8)$ \\
\hline Hypertension & $6(13)$ & $8(16)$ \\
\hline
\end{tabular}




\begin{tabular}{|l|c|c|}
\hline Diabetes mellitus & $1(2)$ & $3(6)$ \\
\hline Rheumatoid or other arthritis & $9(19)$ & $7(15)$ \\
\hline Ischemic heart disease & $2(4)$ & $0(0)$ \\
\hline Calcium supplementation & $16(33)$ & $7(15)^{*}$ \\
\hline Vitamin D supplementation & $23(48)$ & $15(31)$ \\
\hline Antidepressants & $8(17)$ & $7(15)$ \\
\hline Hypoglycaemic agent & $1(2)$ & $3(6)$ \\
\hline Antithrombotic agent (e.g. warfarin, heparin) & $0(0)$ & $1(2)$ \\
\hline
\end{tabular}

Abbreviation: $\mathrm{n}=$ number of participants; $\mathrm{kg}=$ kilogram; $\mathrm{m}=$ metre; IQR = inter-quartile range med $=$ median $; \mathrm{mg}=$ milligram $; \mathrm{ml}=$ milliliter

Note:

$* \mathrm{p}=0.056$

${ }^{a}$ A short validated food questionnaire was utilized to record the calcium intake of certain food items during the past three months [38].

${ }^{b}$ The number of postmenopausal women on current HRT use did not differ between groups $(\mathrm{p}=0.225)$

${ }^{\mathrm{c}}$ The number of standard alcoholic drinks per month for the last 12 month was self-reported. Lifetime smoking calculation $=$ [the average number of cigarettes smoking/day $\mathrm{x}$ years /20] Consistent with exclusion / inclusion criteria, the cohort included one AED-user diagnosed with osteoporosis. 
Table 2: Cross-sectional bone measures

\begin{tabular}{|c|c|c|c|c|c|}
\hline \multirow{2}{*}{$\begin{array}{l}\text { BMD by regions } \\
\left(\mathrm{BMD}=\mathrm{g} / \mathrm{cm}^{2}\right)\end{array}$} & \multirow{2}{*}{ n pairs } & \multicolumn{3}{|c|}{ Mean (SD) } & \multirow{2}{*}{$\begin{array}{c}\text { Mean Within-Pair Difference } \\
\text { (MWPD) as \% of non-users mean } \\
(95 \% \mathrm{CI})\end{array}$} \\
\hline & & AED users & Non-users & $\begin{array}{l}\text { Within-Pair } \\
\text { Difference }\end{array}$ & \\
\hline Total Hip & 48 & $0.933(0.10)$ & $0.971(0.10)$ & $-0.037(0.12)$ & $-3.8(-7.7,-0.1)^{*}$ \\
\hline Neck of femur & 48 & $0.828(0.10)$ & $0.869(0.13)$ & $-0.041(0.12)$ & $-4.7(-8.9,-0.5)^{*}$ \\
\hline Lumbar spine (L2-L4) & 48 & $1.015(0.12)$ & $1.036(0.12)$ & $-0.021(0.14)$ & $-2.0(-0.6,1.9)$ \\
\hline Total forearm & 47 & $0.567(0.06)$ & $0.572(0.05)$ & $-0.005(0.05)$ & $-0.9(-3.3,1.7)$ \\
\hline
\end{tabular}




\begin{tabular}{|c|c|c|c|c|c|}
\hline \multirow{2}{*}{$\begin{array}{l}\text { BMD by regions } \\
\left(B M D=g / \mathrm{cm}^{2}\right)\end{array}$} & \multirow{2}{*}{ n pairs } & \multicolumn{3}{|c|}{ Mean (SD) } & \multirow{2}{*}{$\begin{array}{c}\text { Mean Within-Pair Difference } \\
\text { (MWPD) as \% of non-users mean } \\
(95 \% \mathrm{CI})\end{array}$} \\
\hline & & AED users & Non-users & $\begin{array}{l}\text { Within-Pair } \\
\text { Difference }\end{array}$ & \\
\hline Whole body BMC (grams) & 40 & $2229(237)$ & $2254(246)$ & $-25(246)$ & $-1.1(-4.6,2.4)$ \\
\hline \multicolumn{6}{|l|}{ Follow-up } \\
\hline Trochanter & 48 & $0.710(0.10)$ & $0.745(0.09)$ & $-0.034(0.10)$ & $-4.6(-8.7,-0.4)^{*}$ \\
\hline Inter-trochanter & 48 & $1.072(0.13)$ & $1.101(0.13)$ & $-0.028(0.15)$ & $-2.5(-6.7,1.5)$ \\
\hline Lumbar spine (L2-L4) & 48 & $1.008(0.13)$ & $1.028(0.13)$ & $-0.002(0.14)$ & $-0.2(-5.7,1.8)$ \\
\hline
\end{tabular}




\begin{tabular}{|c|c|c|c|c|c|}
\hline \multirow{2}{*}{$\begin{array}{l}\text { BMD by regions } \\
\left(\mathrm{BMD}=\mathrm{g} / \mathrm{cm}^{2}\right)\end{array}$} & \multirow{2}{*}{ n pairs } & \multicolumn{3}{|c|}{ Mean (SD) } & \multirow{2}{*}{$\begin{array}{c}\text { Mean Within-Pair Difference } \\
\text { (MWPD) as \% of non-users mean } \\
(95 \% \mathrm{CI})\end{array}$} \\
\hline & & AED users & Non-users & $\begin{array}{l}\text { Within-Pair } \\
\text { Difference }\end{array}$ & \\
\hline Whole body & 40 & $1.086(0.09)$ & $1.110(0.08)$ & $-0.024(0.10)$ & $-2.2(-5.0,0.7)$ \\
\hline Whole body BMC (grams) & 40 & $2189(280)$ & $2238(253)$ & $-48.92(281)$ & $-2.2(-6.3,1.8)$ \\
\hline
\end{tabular}

Abbreviations: $\mathrm{BMD}=$ bone mineral density $; \mathrm{BMC}=$ bone mineral content; $\mathrm{SD}=$ standard deviation; $\%=$ percentage; $\mathrm{n}=$ number of participants; $\mathrm{CI}=$ confidence interval

$* p<0.05$.

Note: BMD and BMC were adjusted for age, height and weight prior to analysis. Within-pair difference value was derived from paired t-test (AED user BMD minus non-AED user BMD). MWPD \% of non-AED users mean = MWPD in areal BMD x 100\% / non-AED users mean BMD. A negative value of MWPD \% indicates reduced BMD in AED users compared to non-AED user 
Table 3: Regression analysis for the predictor variable AED therapy duration and the annual rate of bone change

\begin{tabular}{|c|c|c|c|c|}
\hline \multirow[t]{2}{*}{ Areal BMD/BMC ${ }^{\mathrm{a}}$} & $\begin{array}{l}\text { All users } \\
(n=46)\end{array}$ & $\begin{array}{l}\text { "Prolonged users" } \\
(\mathbf{n}=21)\end{array}$ & $\begin{array}{l}\text { Non-users } \\
(n=42)\end{array}$ & \multirow{2}{*}{$\begin{array}{c}\text { Regression Coefficient } \\
(95 \% \mathrm{CI})\end{array}$} \\
\hline & \multicolumn{3}{|c|}{$\%$ Annual bone change of baseline } & \\
\hline Lumbar spine & -0.2 & -0.5 & -0.3 & $-0.008(-0.023,0.008)$ \\
\hline Whole body BMC & -0.2 & -0.3 & -0.2 & $-32.48(-58.28,-6.696)^{*}$ \\
\hline
\end{tabular}

Abbreviations: $\mathrm{BMD}=$ bone mineral density; $\mathrm{BMC}=$ bone mineral content; $\mathrm{SE}=$ standard error; $\%=$ percentage $\mathrm{CI}=$ confidence interval 
$* \mathrm{p}<0.05$

${ }^{a}$ Dependent variable of the multiple regression equation

Note:

The annual rate change in BMD and BMC was adjusted for age, height, and weight prior to analysis.

The independent variables within the model included AED user status, gender, current use of vitamin D supplementation, calcium intake in the past three months (mg/day), menopausal status, current smoking status, concurrent use of multiple AEDs, and AED therapy duration.

Participants currently on hormone replacement therapy were excluded from the analysis.

"Prolonged users" were taking AEDs for $>20$ years (i.e. greater than the cohort mean AED therapy duration) 
Title: Bone loss with antiepileptic drug therapy: a twin and sibling study

\section{Mini Abstract}

Changes in areal bone mineral density (aBMD) and other predictors of bone loss were evaluated in 48 same-sex twin/age-matched sibling pairs discordant for antiepileptic drug (AED) use. AED users had reduced BMD at the hip regions. Prolonged AED users had greater aBMD loss, predicting a higher risk of bone fragility. (50 words) 


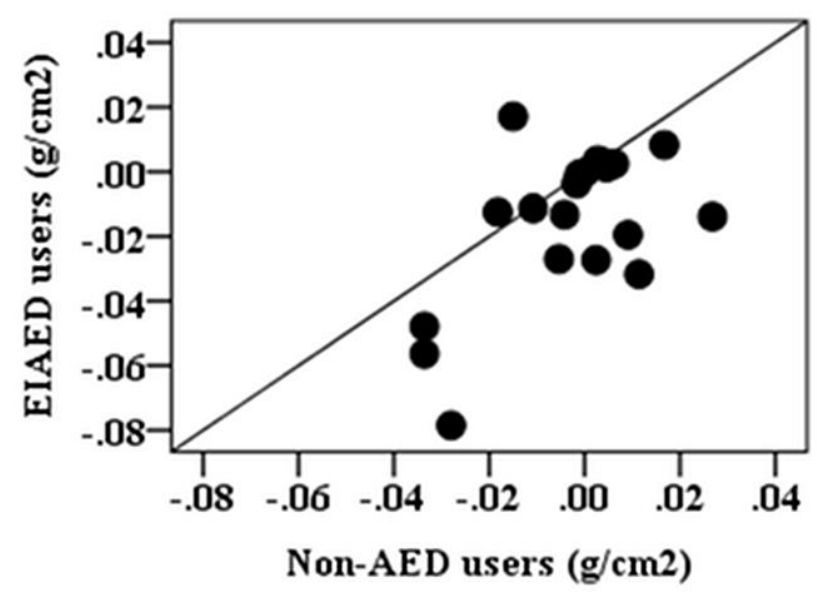

